\title{
Géolinguistique
}

19 | 2019

Varia

\section{À propos des désignations romanes de la taupe}

A proposito delle designazioni romanze della talpa

About Romance Designations of the Mole

Sabina Canobbio, Vittorio Dell'Aquila et Gabriele lannàccaro

\section{OpenEdition}

Journals

Édition électronique

URL : http://journals.openedition.org/geolinguistique/1105

ISSN : 2650-8176

\section{Éditeur}

UGA Éditions/Université Grenoble Alpes

Édition imprimée

ISBN : 978-2-37747-166-9

ISSN : 0761-9081

\section{Référence électronique}

Sabina Canobbio, Vittorio Dell'Aquila et Gabriele lannàccaro, « À propos des désignations romanes de la taupe », Géolinguistique [En ligne], 19 | 2019, mis en ligne le 09 décembre 2019, consulté le 19 décembre 2019. URL : http://journals.openedition.org/geolinguistique/1105

Ce document a été généré automatiquement le 19 décembre 2019.

Géolinguistique 


\title{
À propos des désignations romanes de la taupe
}

\author{
A proposito delle designazioni romanze della talpa \\ About Romance Designations of the Mole
}

Sabina Canobbio, Vittorio Dell'Aquila et Gabriele lannàccaro

1 1. Au moins trois facteurs fondamentaux concourent à l'identification du référent «taupe » (sans spécification ultérieure). Les formes standards correspondantes dans les langues romanes sont les suivantes: it. talpa, port. toupeira, esp. topo, cat. talp, gal. taupa, roum. cîrtiŢă. Pour les formes dialectales nous remercions les comités nationaux du réseau de l'ALiR ${ }^{1}$.

2 Il n'y a pas, du point de vue de la classification, un seul type de taupe : aux Talpidae talpinae correspondent de nombreuses espèces, aux caractéristiques très variées et distribuées dans des aires de répartition différentes ; en Italie on trouve par exemple la Talpa europaea (dans la zone septentrionale et centrale), la Talpa caeca dans les Apennins et les Alpes, et la Talpa romana en Italie centrale et méridionale. C'est surtout à la deuxième espèce qu'il semble que l'on puisse accorder effectivement cette attribution de "cécité ", à cause d'une membrane qu'elle a sur les yeux et que la croyance populaire étend également à d'autres taupes. Mais aussi à cause des poils qui descendent devant les yeux, et surtout, vivant principalement sous terre dans l'obscurité, on finit par retenir qu'elles font rarement usage du sens de la vue.

3 En confrontant la distribution des formes italo-romanes, il y a en effet une certaine coïncidence entre le développement du déterminant « aveugle » et l'aire de diffusion de la Talpa caeca, mais il faut faire attention au fait que cet adjectif, comme nous le verrons, introduit un élément de distinction et de clarification surtout en tant que déterminant du type « souris ». Dans le cas contraire il se vérifierait une superposition, embarrassante pour le locuteur, avec un autre animal qui fréquente le même habitat - la «souris »- qui n'est pas insectivore bien que rongeur et aux habitudes et comportements différents. Si l'on étend l'analyse au domaine roman, les incertitudes et les mélanges entre les espèces peuvent être encore plus importants : par exemple en 
Roumanie au moins deux autres rongeurs sont présents et assimilés à la taupe : le Mus spicilegus et le Spalax leucodon.

4 Ce développement fait référence à l'idée que le locuteur se fait de la taupe et non à une espèce individuelle. En outre, toujours pour l'Italie, même la carte AIS 447 fait référence à la "taupe » en général et les cartes non publiées talpa [v. 4610] e la terra smossa e sollevata dalla talpa come la dite? [v.4606] de l'ALI se réfèrent de manière implicite à la Talpa europaea, mais ont ensuite probablement fini par recueillir, du moins là où celle-ci est présente, les noms de la Talpa caeca. Il convient cependant de signaler que cette - quand bien même nécessaire - simplification comporte en soi quelques risques.

5 On observera en particulier le rapport avec d'autres animaux de la famille des rongeurs muridés, à savoir la souris (Mus musculus) et le rat (différentes sous-espèces de Rattus) ; ou aussi avec des espèces de moindre diffusion comme les hamsters ou les souris des steppes, présents particulièrement en Europe de l'Est. L'enchevêtrement entre les diverses séries dénominatives n'est pas de peu d'importance, comme nous le verrons, pour des raisons de dérivation et d'évolution linguistique, mais aussi parce que cette superposition est favorisée (et au moins dans certaines zones, surtout là où la taupe est moins active) par une sommaire ressemblance physique entre la taupe et certains de ces rongeurs, outre que par la fréquentation substantiellement du même habitat - même si avec des comportements fort différents. Il existe en particulier un animal qui ressemble par la forme du museau à la taupe et qui peut avoir servi d'intermédiaire sémantique : la "musaraigne» (types de Soricidae). Cela est également intéressant car c'est justement sur cet animal (qui est une souris, mais insectivore comme la taupe) que semble s'être développée la plus consistante phase résiduelle de la dénomination latine MUS (pour "souris»), forme qui, comme on le sait, est généralement substituée en Europe latine par d'autres types lexicaux.

6 En outre, la taupe n'est pas aussi universellement répandue et connue: elle est complètement absente en Sardaigne, en Corse et dans une bonne partie de la Sicile, elle n'est pas présente ou moins fréquente dans certaines aires de la péninsule italienne, hypothétiquement en particulier dans celles où un terrain moins favorable rend peu aisées ses activités de fouissement. Ce n'est pas un hasard si dans ces zones la superposition d'identifications et de dénominations avec d'autres animaux est plus fréquente, et en particulier avec la souris.

7 Un autre facteur d'intérêt est donné par le fait que de la latinité elle-même arrivent des signaux non univoques, comme nous le verrons bientôt en nous occupant de la plus grande famille dénominative, qui est composée de noms dérivés de la racine DARB-/ TALP-/TAUP-.

8 2. Parmi les différentes manières possibles de classer les formes, nous avons choisi d'utiliser un critère purement motivationnel, qui procède par augmentation de la transparence synchronique de la forme chez le locuteur : à des formes qui ne sont plus transparentes (ou qui ne l'ont jamais été, si ce n'est à une époque préhistorique) suivent les formes dans lesquelles à la taupe est donné le nom d'un autre animal similaire et ensuite les formes synchroniquement compréhensibles (ou qui de toute façon doivent l'avoir été à une époque pas trop ancienne, étant donné la transparence de la formation) à l'intérieur de la culture qui les exprime. 
9 2.1. Au premier grand groupe appartiennent toutes les dénominations qui dérivent des racines non étymologiquement transparentes. Il s'agit des formes qui ont pour origine la base prélatine *DARB-, les continuateurs du lat. TALP-/TAUP-, qui sont peut-être liés, et celles qui dérivent du germanique *waurp-az. Pour la racine *DARB- (REW 2473 ; FEW 3.13 ; Bertoldi, 1932 ; Franconie, 1986 ; Franconie \& Gassmann, 1987 ; mais déjà attesté en lat., s'il faut croire Walde \& Hoffmann 324 s. v. DARPus, témoigné ensuite à Lyon au $\mathrm{v}^{\mathrm{e}}$ siècle) on soutient l'hypothèse d'un rapport pas trop reculé avec TALPA (par l'intermédiaire des formes rhotacisées) étant donné l'influence de l'évident échange entre les sourdes et les sonores (voir occ. [d'arbu]). D'autres animaux présentent une alternance $d / t$ : on peut voir en particulier le nom de la « grive » (its. dort, it. tordo) en aire alpine dans la riche doxographie de Borghi (2005) (lequel toutefois nie la parenté étymologique entre les formes avec la sourde et celles avec la sonore). Le type *DARBest représenté seulement dans le domaine gallo-roman et est caractérisé par deux phénomènes spécifiques : une alternance initiale $n-/ 3-/ \varnothing$, qui semblerait indiquer une faiblesse du son initial, à considérer sous la lumière d'un possible rapport avec TALP(voir frpr. [dar'bõ] [3ar'bõ]). En outre la base se présente souvent avec le suffixe -ONE ; ce suffixe, partagé dans la forme et dans la fonction par le latin et le gallois, pourrait représenter un passage métonymique de «marque de l'animal » à "animal ", voir par exemple le fr. hérisson du lat. $\operatorname{HERICE}(\mathrm{M})+\mathrm{ONE}$ ou - comme on le verra ci-dessous - la forme alpino-lombarde talpon (TALP- + ONE). C'est précisément à travers la continuité de diffusion du suffixe -ONE que nous pouvons établir un parallélisme important entre les racines *DARB- et TALP- : une aire centrale burgonde qui présente des formes comme darbon s'atténue vers l'extérieur dans des localités qui attestent d'abord tarpon et puis talpon (toutes des formes masculines), jusqu'à des territoires gallo-romans et galloitaliques qui ont taupe/talpa au féminin. Au-delà de cette aire commencent les masculins du type topo (avec la signification en espagnol de "taupe » et en italien de " souris »)2. Un possible lien étymologique de substrat entre les racines est discuté par Bertoldi (1931), qui s'occupe de l'alternance sourde/sonore dans des mots comme pala/ bala « montagne».

10 La forme, attestée dans le Jura [drع'vi] pourrait dériver d'une forme métathétique ancienne *drabaria (voir aussi Franconie \& Grassmann (1987), qui penchent cependant pour une alternance, irrégulière mais fréquente, entre - $r b$ - et - $r v$-, comme dans corbus/ corvus, etc.), que l'on doit reconduire à un *DARB avec suff. -ARIA. Il est possible qu'il s'agisse d'un glissement sémantique de «tanière de la taupe » à « animal "³.

La forme oil [tar'piəِ] pourrait effectivement trouver son origine dans une forme suffixée en -ILLA, mais trois raisons nous font pencher pour -ARIA (et cependant avec un sens du suffixe différent de celui plus fréquent que nous verrons plus bas) : avant tout sa position à la limite entre l'aire oil et l'aire franco-provençale, où [drع'vi] est attesté ; puis pour le -rp-. Enfin cela corrobore l'idée d'un centre burgonde, qui s'étend au nord avec le suffixe -ARIA et au sud avec-ONE.

2.2. TALP- semblerait une dénomination primaire, car elle est présente en latin classique (provenant aussi d'une précédente base prélatine d'étymologie incertaine malgré les nombreuses tentatives faites pour l'expliquer, tentatives résumées dans Walde \& Hoffmann 644 ; pour TALPA voir aussi REW 85.45 ; FEW 12.61 ; DEI 5.3705 ; DELI 4.1310 ; AEI 423). Notre discussion est axée sur le genre grammatical du nom et sur l'alternance irrégulière entre continuateurs de la connexion -LP-. TALPA est masculin à l'époque 
classique et devient féminin à partir de Pline - se différenciant donc d'un nouveau masculin TALPUS (Walde \& Hoffmann 644); nous verrons que cette alternance se poursuit dans les dénominations romanes actuelles ${ }^{4}$.

D'autre part, les formes latines attestées ne sont pas suffisantes pour justifier nombre de résultats phonétiques des variantes romanes. Comme on l'a vu précédemment, certaines zones présentent des formes rhotacisées irrégulières (à savoir dans des aires où $\mathrm{AL}+$ cons. ne donne pas - $\mathrm{AR}-$ ) et une grande partie des variantes dialectales romanes prévoit une forme originaire avec AU et non avec AL (voir ast., its., itc. ['topu], ['topo]). On peut observer par exemple le toscan, où topo ne présuppose pas un lat. TALPUS, mais bien une forme *TAUPUS. C'est précisément l'alternance toscane souris «topo » / taupe «talpa » qui parle en faveur de deux formes sous-jacentes parallèles, bien que corrélées (d'ailleurs, dans l'aire oil et partiellement dans le domaine castillan AL et AU ont les mêmes résultats modernes).

Nous ne nous trouvons cependant pas face à une situation exceptionnelle: des alternances du genre sont déjà connues à l'époque classique, où, en particulier dans la terminologie du monde agricole, sont attestées des variantes urbaines opposées à des variantes rurales, comme dans TABANUS / TAFANUS ou BUBULCUS / BIFULCUS. Nous aurons ainsi des formes urbaines en AL (peut-être centrales), des formes rurales en AU et des formes de contact avec le substrat en AR; nos données ne nous permettent pas de savoir quelle est la forme primaire ; néanmoins, la fréquence du passage $\mathrm{AL}>\mathrm{AU}$ et l'existence de formes en AR nous feraient pencher pour l'attestation classique TALP- - il ne faut cependant pas exclure que la variante avec AL puisse être un hypercorrectisme urbain d'époque romaine.

Nous avons, sur la provision de témoignages classiques et du FEW, recueilli ainsi dans une même grande famille, celle des continuateurs d'un latin classique TALPA / TALPUS, toute la longue et diversifiée série non seulement des formes de genre féminin, mais aussi celles de genre masculin. En fait, alors que pour certains cas on peut probablement déterminer une descendance précise de formes et de séries dénominatives de ce latin tardif masculin *TAUPUS (ainsi par exemple le DCELC sv. topo pour les noms espagnols du type topo, qui, lui, dérive cependant de TALPUS), un regard à une alternance répandue entre formes masculines et féminines et l'évidente conscience de successifs métaplasmes entre un genre et l'autre, toujours possibles, nous font retenir un peu trop rigide et définitive une classification qui prend position, en supposant deux séries complètement distinctes de continuateurs de TALPA et de continuateurs de TALPUS.

16 La classification que nous proposons part ainsi d'une première subdivision basée sur la différence entre les résultats de AL et AU, à leur tour distincts en masculins et féminins ; ces derniers sont considérés ensuite comme absolus, suffixés ou déterminés. En dehors du schéma se posent les formes suffixées en -ARIA pour leur sémantique particulière liée, du moins à l'origine, à la « tanière de la taupe " plutôt qu'à l'animal lui-même.

17 2.3. Le suffixe le plus significatif est sans aucun doute -ōNE qui, comme on l'a déjà observé, ne doit pas être considéré comme augmentatif : il est déjà un suffixe indoeuropéen pour la formation de classes nominales et amplement employé dans cette fonction depuis le latin. Dans les langues romanes il a une valeur génériquement altérative - si toutefois il est appliqué à des bases déjà romanes - qui souvent prend une fonction de diminutif (comme en catalan, en occitan ou en lombard alpin) et c'est 
seulement dans l'aire italo-romane qu'il acquiert des acceptions augmentatives. Les talpon, tout comme les darbon, ne sont donc que des «taupes" tout court. Les vraies suffixations à l'origine diminutives sont celles en -INU, -ILLU, -ITTU, répandues dans l'aire ibérique et de la Haute-Italie (voir cast. [to'pino], [to'piאo], [to'pito]; its. [tu'pin]). 2.3.1. Le déterminant le plus répandu, comme l'on peut s'y attendre, est l'adjectif " aveugle ", attribué en particulier à la base "souris ». Sa particulière diffusion dans l'aire italo-romane est donc due à la nécessité de distinguer deux animaux de même étymologie mais différents de par la spécification de l'une des caractéristiques prédominantes de l'un de ceux-ci (on se rappellera que l'Italie centrale et l'Italie méridionale constituent précisément l'aire de diffusion de la Talpa caeca). Le côtoiement de "taupe" et "souris" peut avoir joué un rôle important dans la perception des locuteurs de cet animal, ayant à peu près l'apparence de la "souris ". Ceci renforce l'hypothèse d'une différenciation ancienne entre formes urbaines et rurales entendues comme ayant le même radical: en aire urbaine nous pouvons supposer que la différence entre les résultats de TALPA et de TAUPA (emprunt rural) ait été suffisante pour distinguer les deux animaux, alors qu'en aire rurale le fléchissement des deux formes sous $\mathrm{AU}(>0)$ a créé la nécessité d'une redétermination. En Toscane, à cette dyade s'ajoute un élément, talpone, signifiant " rat ».

2.3.2. Un autre déterminant part de la motivation de «fouiller du groin » / «fouir » : il s'agit de l'its. ['topa ru'gaja]. L'acte de fouissement est déterminant dans la perception populaire de la taupe, comme l'est le petit tas de terre qui caractérise sa tanière: certaines dénominations de l'animal sont donc en réalité transposées à partir du nom

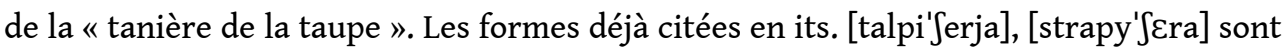
en effet relatives au terrier, soit, étymologiquement, à « ce que creuse la taupe » : c'est au fond le même rapport qui lie le nom du lapin (lat. cuniculu(M)) à sa tanière, dans de nombreuses variantes romanes.

2.4. Nous pouvons ensuite reconstruire une série de trois différents types dénominatifs métonymiques, fruit du passage sémantique du signe que l'animal laisse dans le terrain à l'animal lui-même : la "tanière de la taupe », le "petit tas de terre produit par la taupe " et la "terre jetée à l'extérieur de la tanière par la taupe " (pour ces deux derniers voir ci-dessous). Ces identifications ont lieu en vertu du fait que l'animal est souvent repéré par le paysan grâce à cette activité d'excavation (ou par les dégâts qui en découlent) plutôt que par une rencontre directe. Nous verrons plus loin des dénominations transparentes liées aux activités de l'animal ou aux résultats de telles activités.

21 La première forme que nous considérons est celle au suffixe simple -ARIA (type *TAlPARIa / TAUPARIA), qui continue en port. Toupeira; la deuxième, répandue en aire alpine méridionale, semblerait partir d'un *TALPICEARIA / TAUPICEARIA (voir en its. Talpiscera / trapuscera) : dans ce cas, le même suffixe -ARIA est précédé d'une forme altérative *TALPICEA - des résultats de ce même suffixe comme diminutif/affectif sont répandus dans l'aire italo-romane comme -izza / -iccia (its. -iscia). Le troisième résultat est talpinera, basé sur *TALP-IN-ARIA, qui pourrait avoir été influencé par une réétymologisation sur "taupe noire / souris noire» (voir en itc. [topa'nira]), pour laquelle on peut penser à une étymologie populaire.

22 Ensuite, on peut faire remonter le fpr. ['vorpa] à une base germanique *waurps, liée à la même racine que le verbe allemand werfen ( $<$ warpjan « jeter $)$. Cette forme est 
répandue dans différentes langues germaniques précisément avec le sens de "taupe " (voir par exemple le bas saxon mulworp). Toutefois les formes attestées sont presque exclusivement composées avec ${ }^{*} m \bar{u} l-$, forme superficielle composée de trois racines possibles ayant le sens de " tas », « terre », « moudre » (voir par exemple l'it. mulino). La même racine se trouve dans le fr. mulot, voir infra. La phonétique de ['vorpa] nous fait pencher pour un emprunt de substrat, peut-être burgond vu la disposition géographique, et non pour un emprunt germanique récent, parce que toutes les formes alémaniques environnantes présentent le passage régulier $p>f$ de la $\mathrm{II}^{\mathrm{e}}$ rotation consonantique. Éventuellement, suivant le FEW 3.612, on pourrait penser à une réétymologisation tardive sur le lat. VǓLPES.

3. Au second grand groupe appartiennent toutes les dénominations qui dérivent de noms d'autres animaux. Les sous-groupes principaux sont au nombre de trois: les emprunts du mot "taupe» dans d'autres langues, les formes dénominatives qui naissent $\mathrm{du}$ côtoiement dans une certaine mesure transparente - à savoir intragénétique - de la taupe avec d'autres animaux et enfin les formes qui associent les deux types, c'est-à-dire des emprunts de mots qui dans d'autres langues indiquent des animaux semblables à la taupe.

Des formes arrivées par adstrat qui se réfèrent à la taupe considérée dans d'autres traditions et langues: surtout le fr. mulot, qui, comme on l'a vu ci-dessus, est un emprunt au germanique *mül-; l'éminence, dans l'aire balkanique, du type cîrtiţăa, emprunt sud slave (<blg. КъРТИЦА «taupe ", à son tour diminutif de asl. КъРтъ); une variante en est le fri. chert du slovène krt. Et toujours à l'intérieur de ce sous-groupe nous trouvons des dénominations comme le roumain ['vokont]], ['vakand], à relier au hongrois vakond (Spalax leucodon), sorte de taupe particulièrement répandue en Europe centrale et orientale.

D'autres formes utilisent le nom d'autres animaux pour indiquer la taupe ; tout d'abord le groupe de noms originairement de la souris, qui a une parenté étymologique comme on l'a vu plus haut, et celles qui ont pour origine un rapprochement avec des animaux différents comme le loir, la zibeline et peut-être aussi le cochon.

Dans un premier cas nous avons les continuateurs du lat. Mūs, en particulier avec un (compréhensible) suffixe augmentatif -ONE (its. [my'suy]) ou l'habituel déterminant "aveugle» (comme en its. ['musek], ['muzega]). La dérivation du nominatif est cependant remarquable et pourrait faire penser, étant donné la position orientale des voix dans le domaine, à une possible connexion avec aat. mūs (all. Maus « souris»), par l'intermédiaire de dialectes alémaniques ou bavarois. On peut toutefois invoquer d'autres raisons : l'ancienneté du composé et surtout des motifs de personnification à valeur totémique; comme on le sait, les nominatifs latins se perpétuent dans les langues romanes comme nomina agentis - par définition, donc, applicables à des éléments à haute agentivité.

Nous avons ensuite les nombreux continuateurs (tous issus d'aire italienne) du lat. SORǏCE(M) (REW 8098 ; FEW 12.110; DEI 5.3556), d'où le fr. souris (*SORİCE, fém.) et l'italien sorcio (*SORICIU) « souris ». Les formes sont soit masculines (itm. ['surətfə]) - les plus fréquentes - soit féminines (itm. [sura'tfeja], [suri'tfu:la], avec des suffixes), et, si ce n'est dans une attestation de la Campanie, suffixées ou encore plus souvent accompagnées de (nécessaires) déterminants ou différenciateurs du type « aveugle » ou « de terre ». Enfin le type supposé *sorcenARIA est d'un intérêt incontestable parce qu'il 
semblerait formé par les mêmes modalités que *TALPINARIA. Dans la forme attestée en itm. [fortfe'nara] il y a une fricative labiodentale sourde $f$ - qui crée quelque perplexité : il pourrait cependant s'agir d'un cas de reconstruction incongrue (contamination d'étymologie populaire avec "fort ", à cause de l'efficience excavatrice de la taupe ?). Précisément dans l'ALI - sur ce point - il y a d'autres preuves d'initiales faibles qui tendent à s'atténuer ou tomber.

On trouve aussi des dénominations du type « rat » (dont l'origine est controversée : du lat. RĂPIDU(M); Walde \& Hoffmann: «raubend, reißend, heftig» ou d'un thème onomatopéique $r-t$; voir REW 7089 ; DEI 5.3212 ; DELI 4.1036; AEI 348) présentes en particulier dans l'aire ibérique, mais aussi dans celle gallo-romane (voir le frpr. ['rata], le cast. [r:a'ton], le port. ['r:ate]). Dans de nombreux cas il y a une palette de déterminants (" aveugle »; " de terre », « des champs », etc.) qui tentent d'assainir les possibles ambiguïtés rat / souris ou rat / taupe (voir le port. ['r:atu 'se $\mathrm{du}$ ] ; l'itc. ['sorka 'tfeka]; le port. ['r:ate uf 'kẽpuf]). Les formes ibériques sont également fort intéressantes, elles croisent «rat » avec «souris » comme le port. ['r:atu to'peru] ou ['r:atu to'pinu], mais aussi l'its. [rat tu'piy] qui représente une assez grande série d'attestations présentes dans le lombard alpin avec des formes fréquentes du type [ratu 'pon] (même dans Cherubini [1839, vol. $4: 16]$ nous trouvons pour le milanais rat topon «taupe »). Ensuite, on remarquera le composé occitan ['rata də 'bufa], à savoir vraisemblablement « rat qui souffle »; nous en verrons les liens avec le thème -buff.

Certaines formes italiennes et roumaines sont en filiation directe avec MUS ARANEA " musaraigne ", composé attesté déjà en latin et qui donne des continuateurs du type mosgagna, mosegagno, musaragn, qui se réfèrent, selon les zones, à la «taupe » ou à la «musaraigne». De manière semblable le cast. [li'ron] du lat. GLIRE(M) «loir» (REW 3787) plus le suffixe -ONE.

4. Le troisième regroupement est composé d'emprunts hétérogénétiques : tout d'abord ceux dérivés de noms qui se réfèrent originairement à la souris, comme l'its. ['lok], du celtique *lukotis (breton logod, gaélique luch «souris»); ici on trouve aussi le type roumain guz, guzu et dérivés, qui reprend le hongrois güzü (Mus spicilegus, « souris des steppes ", espèce répandue uniquement en Europe de l'Est). En outre, la forme grico

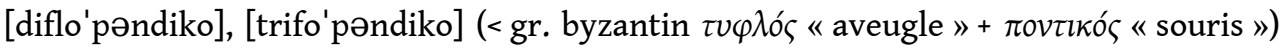
est particulière (peut-être à attribuer au premier regroupement) qui reprend dans le composé des associations communes dans l'aire romane environnante.

31 D'autres rongeurs sont représentés par le type moldave [so'bol], emprunt est-slave d'une racine qui en russe donne соболь «zibeline» (et ici il faudra observer que le même terme «zibeline » est en français un emprunt aux langues slaves). Il est possible que même la forme istrienne ['t fobo] soit à mettre en relation avec ce thème.

Parmi les géosynonymes roumains pour "taupe » attestés dans les dictionnaires nous trouvons mitorlan (avec le sens dans certaines zones de « chat») : la carte ALIR ROU 54 atteste cependant [mitsor'lan] avec palatalisation $t>t s$ irrégulière devant $-o$ ]. Quant

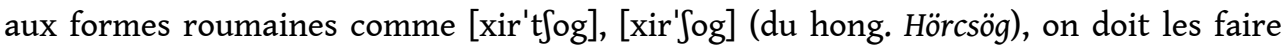
remonter à une superposition avec " hamster " : en Europe centrale le hamster est fort répandu et vit à l'état sauvage. Plus éloignée et douteuse semble être l'association avec le cochon (en fri. farc), voix du reste très répandue dans l'aire: il s'agirait d'un longobard * $f_{f a r h}(a)$, correspondant phonétiquement au lat. PoRcus «porc» (voir all. 
Ferkel « cochon de lait »). Le passage est probablement dû à l'action de «fouiller du groin » (Cortelazzo \& Marcato, 2005 : 192 ; Pellegrini, 1983).

5. D'autres dénominations, désormais pleinement transparentes, qui s'entrecoupent en partie avec les précédentes (voir «fouiller du groin», «fouir »), partent des activités typiques de la taupe, ou du résultat de celles-ci. Nous avons ainsi des formes dérivées du lat. RUMARE « fouiller, fouiller du groin » en frioulan (['rumula]) et en gallo-roman (éventuellement composée avec «terre»); ou, comme dans l'its. [ryga'tera] dont le déterminant est "fouiller» («déranger», voir REW 2907 ERUCA), ici aussi en composition avec «terre ». Au Portugal les formes du lat. (EX) CAVARE sont communes, toujours composées avec «terre» ([keße'ter: e] et similaires, ou, en italo-roman avec « terrain » ou « décapeuse » (itm. [kava 'rofpa]). Toujours au « fouissement » se réfèrent les formes du lat. FODICARE « creuser», soit pures (its. ['fodga]) soit suffixées en -ONE, communes dans le domaine d'oïl et en wallon ([fấ'jõ]]). Une autre motivation est celle de "soulever " (clairement les mottes de terre), comme en lad. et its. ['solva] (déverbal de $<$ lat. SUBLEVARE, REW 8373).

D'origines non latines viennent ensuite le port. ['bufo] et autres dérivés, vraisemblablement d'une base buff (REW 1373), en rapport à l'idée de « souffler » (scil. la terre à l'extérieur du trou); les formes françaises septentrionales ['mut] (éventuellement avec suffixe), dérivées de -mott (REW 5702) "petite montagne de terre ", et les types, parements français, d'attribution plus incertaine, ['mu] (lat. MUTARE) et ['by] (BUCA ?). Ce dernier pourrait être mis en relation avec oïl [bus'rø] (d'un latin *BUCEAROLA, forme suffixée de *buceu > lomb. bus (VSI 2.555-556) et le type galloroman ['bulego] (métahèse de buculu(m), voir lomb. böc' (DEEG 326) ? En Roumanie est également attesté un [łor'lan], dérivé du verbe a ghiorai "grogner, fouiller du groin, faire un vilain bruit ».

6. Le nom de la taupe peut aussi dériver de ses caractéristiques physiques: en particulier en Roumanie, le déterminant "vue limitée » est à la base de la forme [wor 'bete] (< ORBUS, avec suffixe); de couleur sombre, aux dimensions réduites et au statut « caché » de l'animal se réfère [fi'tfor de 'popə] « fils de prêtre ».

7. D'étymologie obscure est la forme d'Italie centrale [ka'topa] (composé gréco-latin katatopa « souris qui est au-dessous »?).

\section{BIBLIOGRAPHIE}

AEI = DEvoTo Giacomo, 1968, Avviamento alla etimologia italiana, Florence, Le Monnier.

ALI = ISTITUTO DELL'ATLANTE LINGUISTICO ITALIANO, Atlante Linguistico Italiano, 1995-, Rome, Istituto Poligrafico e Zecca dello Stato.

ALiR = Atlas linguistique roman, 1996-, Rome, Istituto Poligrafico e Zecca dello Stato.

AIS = JABERG Karl \& JUD Jakob, 1927, Sprach- und Sachatlas Italiens und der Südschweiz, Zofingue, Rieger \& Co., 1928-1940. 
BERTOLDI Vittorio, 1931, «Problèmes de substrat : essai de méthodologie dans le domaine préhistorique de la toponymie et du vocabulaire », Bulletin de la Société de linguistique, $\mathrm{n}^{\circ} 32$, p. 93-184.

BORGHI Guido, 2005, «*drexo-, *dresgo-, *dresdo- “tordo" e loro corrispondenti non romanzi: dialettologia indoeuropea preistorica sparsa nelle dialettologie moderne », dans M. Pfister et G. Antonioli (éds), Itinerari linguistici alpini: Atti del Convegno di dialettologia in onore del prof. Remo Bracchi, Tirano - Grosio, Istituto di Dialettologia e di Etnografia Valtellinese e Valchiavennasca, p. 205-236.

CHERUBINI Francesco, 1839, Vocabolario Milanese-Italiano, Milan, Dall'Imperial Regia stamperia.

Cortelazzo Manlio \& Marcato Carla, 2005, Dizionario etimologico dei dialetti italiani, Turin, UTET.

DEEG = ANTONIOLI Gabriele, BRACCHI Remo \& RINALDI Giacomo, 2012, Dizionario etimologico-etnografico grosino, Sondrio, IDEVV.

DCELC = COROMINAS Joan, 1955, Diccionario crítico etimológico de la lengua castellana, Madrid, Editorial Gredos / Berne, A. Francke AG.

DEI = BATTISTI Carlo \& ALESSIo Giovanni, 1966, Dizionario etimologico italiano, Florence, G. Barbèra.

DELI = CORTELAZzo Manlio \& ZoLli Paolo, 1979-1988, Dizionario etimologico della lingua italiana, Bologne, Zanichelli.

FEW = WARTBURG Walther vON et al., 1922-2002, Französisches etymologisches Wörterbuch. Eine Darstellung des galloromanischen Sprachschatzes, Bonn, Klopp / Heidelberg, Winter / Leipzig-Berlin, Teubner / Bâle, Zbinden, 25 vol.

FRANCONIE Hélène, 1986, « Les bases BUFF-, TALP- et DARB- dans les dénominations de la taupe en gallo-roman », Géolinguistique, nº 2, p. 199-235.

FRANCONIE Hélène \& GASSMANN Heinz, 1987, « Les désignations de la taupe et de la taupinière en Suisse romande ", Géolinguistique, n 3, p. 161-17.

Pellegrini Giovan Battista, 1982, « Postille onomasiologiche friulane e alpine », Ce Fastu?, n 58, p. 95-108.

REW = MEYER-LÜBKE Wilhelm, 1930-1935, Romanisches etymologisches Wörterbuch, Heidelberg, Winter.

TAMINE Michel, 1992, Dictionnaire du français régional des Ardennes, Paris, Éditions Bonneton.

VSI = Vocabolario dei dialetti della Svizzera italiana, 1952-, Lugano, Natale Mazzucconi / Bellinzona, Centro di dialettologia e etnografia.

WALDE Alois \& HOFMANN Johann Baptist, 1938-1954, Lateinisches etymologisches Wörterbuch, Heidelberg, Carl Winter.

\section{NOTES}

1. Les indications nationales ont été extrapolées à partir des atlas linguistiques nationaux des différents domaines linguistiques romans. Pour une liste complète des atlas, voir AliR, vol. II/c, 2019 (2 vol.). Nous remercions aussi Jean-Pierre Lai pour la révision du texte en français.

2. Voir Franconie (1986). 
3. Voir avec suffixe identique les formes du type * TALPICEARIA et *TALPINARIA (voir its. [talpi'Serja],

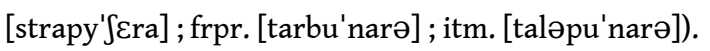

4. La racine est aussi à mettre en relation avec le lat. TELLUS « terre ».

\section{RÉSUMÉS}

L'objectif de cet article est de montrer la diffusion des dénominations de «taupe " dans le domaine linguistique roman. L'approche méthodologique est principalement de type onomasiologique, mais les parentés étymologiques formelles sont aussi prises en considération. Le résultat le plus remarquable de la recherche est la mise en évidence du rapport dialectique au niveau diatopique entre les aboutissements modernes d'une forme centrale prélatine *darb et les formes périphériques abouties du latin rurale (ou des langues italiques anciennes) TAUPA.

Lo scopo di questo articolo è di mostrare la diffusione delle denominazioni della «talpa» nel dominio linguistico romanzo. L'approccio metodologico è principalmente di tipo onomasiologico, ma vengono prese in considerazione anche relazioni etimologiche formali. Il risultato più notevole della ricerca è proprio la relazione dialettica a livello diatopico tra i risultati moderni di una forma centrale prelatina $*$ darb e forme periferiche derivate del latino rurale (o da antiche lingue italiche) TAUPA.

The aim of this paper is to show the geographical distribution of the names of the "mole" in the Romance dialects. The methodological approach is primarily onomasiological, but formal etymological issues are also taken into consideration. Noteworthy is the dialectical relationship at a diatopic level between the modern outcomes of a central prelatine form *darb and the peripheral forms of rural Latin (or ancient italic) TAUPA.

\section{INDEX}

Parole chiave : talpa, dialetti romanzi, onomasologia

Keywords : mole, Roman dialects, onomasiology

Mots-clés : taupe, dialectes romans, onomasiologie

\section{AUTEURS}

SABINA CANOBBIO

Università degli studi di Torino

\section{VITTORIO DELL'AQUILA}

Centre d'études linguistiques pour l'Europe (Milan)

\section{GABRIELE IANNÀCCARO}

Università di Milano-Bicocca 